\title{
Philonsorbonne
}

3 | 2009

Année 2008-2009

\section{Le retour de la physique dans la Métaphysique d'Aristote}

Annick JAULIN

\section{OpenEdition}

1 Journals

Édition électronique

URL : https://journals.openedition.org/philonsorbonne/233

DOI : 10.4000/philonsorbonne.233

ISSN : 2270-7336

Éditeur

Publications de la Sorbonne

Édition imprimée

Date de publication : 15 avril 2009

Pagination : $97-107$

ISBN : 978-2-85944-624-6

ISSN : 1255-183X

Référence électronique

Annick JAULIN, « Le retour de la physique dans la Métaphysique d'Aristote », Philonsorbonne [En ligne], 3 | 2009, mis en ligne le 30 janvier 2013, consulté le 08 juin 2021. URL : http://

journals.openedition.org/philonsorbonne/233; DOI : https://doi.org/10.4000/philonsorbonne.233

(c) Tous droits réservés 


\title{
Le retour de la physique dans la Métaphysique d'Aristote ${ }^{1}$
}

\author{
Annick Jaulin
}

\section{Du titre de cette conférence}

Le titre de cette conférence a un sens tout relatif à l'édition des œuvres d'Aristote, puisqu'il faut attendre la constitution du corpus proposée par Andronicos au premier siècle avant J.-C., pour que le terme de métaphysique fasse référence au rassemblement des textes que nous possédons. Ce titre n'aurait pas eu grande signification pour Aristote.

Si l'on envisage maintenant le contenu des traités, en négligeant la question de l'édition du texte, ce titre est, une fois encore, assez malheureux, car une opération de traduction terme à terme de chacun des deux termes «physique » et «métaphysique » dans le lexique aristotélicien, reviendrait à prendre pour objet « le retour de la philosophie seconde dans la philosophie première ». Cet énoncé ne veut strictement rien dire, puisque la philosophie première est ainsi nommée par Aristote pour avoir dépassé et englobé la philosophie seconde ou «physique » qui ne saurait faire un retour en un lieu qu'elle n'a jamais connu et qui est désormais délimité ou défini par des unités de mesure qu'elle n'a pu produire.

Pourquoi donc avoir choisi un si mauvais titre quand nulle contrainte n'était imposée ? C'est qu'il y a bien un sens à parler du retour de la physique dans la Métaphysique d'Aristote, si l'on développe ce titre de la manière suivante : dans ses traités de philosophie première, transmis sous l'intitulé de Métaphysique, Aristote fonde et justifie la réforme nécessaire de la révolution philosophique antérieure ou de la philosophie première qui en était issue, la philosophie première platonicienne, en montrant qu'elle ne

1. Ce texte est la reprise d'une conférence prononcée le 13 février 2008 dans le cadre du séminaire Travaux en cours, organisé par B. Binoche et Ch. Jaquet, à l'UFR de Philosophie de l'Université Paris1-Panthéon-Sorbonne. 
peut entièrement se substituer à la philosophie qui l'a précédée, à savoir les théories des principes et des causes élaborées par les physiologues, nommés aussi «physiciens». Si l'on conserve l'identité entre le terme «métaphysique», inventé par les éditeurs d'Aristote, et la philosophie première aristotélicienne (on a montré ailleurs ${ }^{2}$ l'équivalence entre «philosophie première », « sagesse » et «science de l'être en tant qu'être »), on doit entendre le retour de la physique comme le retour de ce que la forme antérieure de la désormais philosophie première, la philosophie platonicienne, aurait voulu dépasser sans y parvenir, à savoir les théories physiques concernant les causes. Ce retour concerne notamment la cause motrice.

Bref, ce titre expose comment la version aristotélicienne de la philosophie première se distingue de la version platonicienne de la même philosophie par la nécessité de reconnaître l'importance de la cause motrice, ou par l'impossibilité (dont la démonstration occupe une grande partie des traités) de réduire la causalité à la causalité formelle. Les traités métaphysiques d'Aristote sont donc à la fois la reconnaissance ou l'assomption de la révolution socratico-platonicienne dans la théorie des causes, et la constatation critique que la cause formelle ne suffit pas à rendre raison de ce qui est, qu'il importe en conséquence de proposer une nouvelle formulation de la théorie des causes, dans laquelle la causalité motrice et la causalité formelle pourront être associées, ce qui se produit en effet dans la théorie de la cause finale. Ce retour de la cause motrice dans la philosophie première aristotélicienne mesure la différence entre la philosophie première d'Aristote et celle de Platon. Notre titre a donc du sens seulement si l'on établit un lien entre philosophie première et philosophie platonicienne des formes, relativement à laquelle le «retour» de la physique a quelque signification. Le platonisme apparaît dès lors comme la philosophie qui tend à mettre la cause motrice entre parenthèses, ce dont Plotin donnera l'expression la plus achevée. Le «retour de la physique dans la métaphysique d'Aristote » est la manière précise de désigner la philosophie première aristotélicienne comme réforme de la philosophie première platonicienne ; il y a, autrement dit, une équivalence entre la réinsertion de la causalité motrice dans la théorie des causes et la transformation de la théorie des formes.

\section{Retour de la physique et problèmes philologiques}

Avant de développer les implications philosophiques de cette réforme de la philosophie première, je voudrais d'abord indiquer comment cette perspective, encore programmatique dans la description que nous venons

2. Voir A. Jaulin, «Introduction », in Aristote, Métaphysique, Paris, Garnier-Flammarion, 2008, p. 16-33; «La philosophie première dans le livre G », in Aristote Métaphysique gamma, Édition, traduction, Études, Louvain-Paris-Dudley, Peeters, 2008. 
d'en présenter, est de nature à intervenir dans la position et le traitement de certains problèmes philologiques. Ce qui est décrit comme un « retour de la physique » a une incidence directe sur le statut d'authenticité disputée de certains livres des traités métaphysiques et notamment sur le statut du livre L, mais pas uniquement, puisque le statut du livre a aussi est concerné, voire celui du livre K ; bref, les trois livres qui, en dehors de D, suscitent les soupçons sur leur appartenance aux traités métaphysiques, à cause d'un rattachement à des questions physiques.

Quelles que soient les généalogies fictives proposées, le statut du livre L n'a cessé de faire problème pour les interprètes des traités métaphysiques qui l'ont généralement considéré comme un livre de jeunesse, pour des raisons diamétralement opposées. On connaît la position ancienne de Jaeger, fondée sur le schème psychologique du roman de formation, où le jeune Aristote aurait écrit ce traité théologique dans sa jeunesse platonicienne. Plus récemment un interprète italien, Pierluigi Donini, en accord avec les analyses de M. Frede qui affirme le statut indépendant du livre $\mathrm{L}$ parmi les traités métaphysiques au point d'adopter comme règle méthodologique de le lire sans tenir compte des autres traités métaphysiques ${ }^{3}$, situera également ce traité comme un traité de jeunesse, mais parce qu'Aristote considèrerait la physique comme science universelle et n'aurait pas encore découvert la métaphysique comme une science distincte de la physique ${ }^{4}$. Sans entrer dans les hypothèses gratuites, contradictoires et infondées sur les pensées de jeunesse d'Aristote, on retiendra que, pour la plupart des interprètes ${ }^{5}$, le livre L, pour des raisons opposées (mais, au fond peut-être identiques), fait tache au milieu des traités métaphysiques.

Il semble que ce que l'on peut caractériser comme « un retour de la physique » supprime l'aspect aberrant de la présence de ce livre dans les traités métaphysiques: L est le livre qui donne une théorie complète des causes, en insistant sur l'importance de la cause motrice, notamment au chap. 5 , où il est montré que l'on ne saurait se contenter des causes internes (matière, forme et /ou fin). En tant que tel, sa présence est nécessaire dans les traités métaphysiques, car il est le seul livre des traités qui donne un traitement positif de la cause motrice. Sans lui, la position d'Aristote en matière de philosophie première ne pourrait tout simplement pas être connue et évaluée, car, pour le dire de manière synthétique, on ne saurait pas, sans L comment ce qui semble être un élément nécessaire de l'explication du mouvement, à savoir la thèse nécessaire d'une substance immobile, constitue une théorie des moteurs immobiles que l'on ne saurait d'ailleurs réduire au premier d'entre eux.

3. Ce point de vue est exprimé à plusieurs reprises dans M. Frede and D. Charles, Aristotle's Metaphysics Lambda, Oxford, Clarendon Press, 2000 ; par exemple, p. 4, 53.

4. P. Donini, La metafisica di Aristotele, Roma, Carocci, 2007 (1995 ), p. 158.

5. La position de M. Frede représente la position qui fait actuellement consensus. Elle ne comporte pas d'hypothèses génétiques, mais renvoie au fait que l'édition d'Andronicos rassemble des traités indépendants. 
Le livre L est donc l'illustration par excellence d'un problème qui n'a cessé de préoccuper et de gêner les interprètes des traités, celui du lien entre physique et métaphysique. Dans ma thèse ${ }^{6}$, j'avais noté, à la suite de Ross, qu'Aristote faisait « peu d'efforts pour maintenir distincts les domaines de la physique et de la métaphysique » et que les commentateurs qui voulaient établir une stricte séparation entre les deux ne tenaient aucun compte de l'importance des arguments physiques dans un grand nombre de livres des traités métaphysiques, notamment en G 5, lorsque, dans l'exercice de persuasion des physiciens, Aristote proposait une théorie renouvelée du mouvement. Le propos concerne le projet même des traités : une théorie renouvelée de la cause motrice, qui implique une critique de la version qui en était proposée par les physiologues, est une part nécessaire de la critique de la théorie platonicienne des formes, dont une des incapacités fondamentales est celle de rendre compte de la génération et de la corruption des substances sensibles. Si la théorie de la forme comme synthèse (théorie de la cause formelle) est le dépassement fondé de la théorie des principes et des causes comme éléments constitutifs, en quoi consistait l'ancienne théorie insuffisante des physiologues, la théorie des moteurs, avec la distinction des moteurs mus et des moteurs immobiles, est un complément tout aussi nécessaire à la théorie des formes qui ne se mettent pas d'elles-mêmes en mouvement.

L ne constitue donc pas un traité que l'on pourrait lire séparément des autres traités métaphysiques, pas plus que le livre a n'est un livre égaré dans ces traités, alors qu'il aurait été destiné initialement à servir d'introduction à un traité de physique ${ }^{7}$. Car, si c'était le cas, que devrait-on faire alors de G 5, de Z 7-9, de H 2-5, du livre Q en son entier ? Que devrait-on surtout faire d'assertions, comme celle de Z 11 (1037a 13-17) qui manifeste que la définition des substances sensibles est un des buts de la recherche conduite dans les traités :

«En effet, c'est dans ce but que nous essayons d'établir des définitions aussi à propos des substances sensibles, bien que, en un certain sens, l'étude des substances sensibles soit la tâche de la physique c'est-à-dire de la [15] philosophie seconde, car le physicien ne doit pas seulement acquérir des connaissances sur la matière, mais aussi, et même davantage, sur la <substance> selon l'énoncé »,

ce qui n'étonne pas vraiment qui se souvient de la remarque critique, même un peu attristée, livrée en A 9 (992a 25), lors de la présentation de la doctrine platonicienne des formes :

«D'une façon générale, alors que la sagesse recherche [25] la cause des êtres visibles, nous l'avons laissée de côté (car nous ne disons rien de la cause d'où vient le principe du changement) et, en croyant énoncer leur substance,

6. A. Jaulin, Eidos et Ousia. De l'unité théorique de la Métaphysique d'Aristote, Paris, Klincksieck, 1999, § 42, p. 77.

7. Voir op. cit., note 1, p. 10-11. 
nous affirmons l'existence d'autres substances, mais pour dire comment celles-ci sont substances des êtres visibles, nos explications sont creuses, car « participer», comme nous l'avons dit aussi auparavant, ne veut rien dire »,

critique qui se termine par le constat que : «l'examen de la nature est totalement ruiné ».

On comprend sans peine que si l'on ne veut pas ruiner l'étude de la nature, il faut modifier la théorie platonicienne des causes, or l'apport platonicien original à la théorie des causes consiste dans la cause formelle (puisque cause matérielle et cause motrice étaient déjà connues avant les Pythagoriciens, selon les descriptions du livre A), il faut donc modifier la théorie platonicienne des formes. Ce sera chose faite quand, par la théorie de la cause finale, la cause formelle des platoniciens et la cause motrice des physiciens auront été conciliées : la forme étant l'être en acte d'une chose, son état accompli, est la fin d'un processus positif qui se nomme mouvement. Penser la forme comme fin, c'est la penser comme le moteur immobile d'un mouvement qui est un processus accompli par des moteurs mus ou intermédiaires.

Inscrire dans ce cadre le débat mené dans les traités métaphysiques est extraire la confrontation entre les deux philosophies premières de la Grèce classique de la problématique de l'empirisme versus idéalisme, qui n'a strictement aucune pertinence pour en rendre compte, car, si l'on veut donner un sens philosophique qui ne soit pas en même temps un simple anachronisme au tableau de Raphaël, la différence entre le haut et le bas n'est pas quelque différence de sublimité à laquelle nous a habitués une pieuse précipitation spéculative, mais tout simplement la différence entre le haut et le bas du genre en laquelle consiste la divergence dans la conception des universels entre Platon et Aristote. Il est vrai que la divergence met en jeu les conceptions de l'être et de l'un, ce qui n'est pas rien.

\section{Retour de la physique et problèmes philosophiques}

Si l'on envisage maintenant le retour de la physique dans les traités métaphysiques du point de vue des problématiques philosophiques, on pourrait caractériser ce retour par le changement de contenu, de sens et de statut qui affecte la substance immobile. Qu'il y ait une substance ou une nature immobile (l'équivalence entre les termes de substance et de nature est déjà d'elle-même significative) c'est ce que G 5 cherche à mettre en évidence pour en persuader les physiciens, enclins à affirmer la co-présence des contraires (1010a 33-36). On les en persuadera d'ailleurs par un argument radical : affirmer la co-présence des contraires, est affirmer le repos plus que le mouvement, ou mieux l'identité des deux, car, si tous les contraires sont donnés en même temps, il n'y a pas de «vers quoi » un mouvement pourrait avoir lieu; affirmer la co-présence des contraires est 
donc nier le mouvement. Mais la nécessité de cette substance immobile étant reconnue, on ne pourra plus la faire consister dans les formes ou les nombres, candidats que l'école platonicienne proposait dans cette fonction, comme le rappellera le début du livre $\mathrm{L}$.

On remarquera, au passage, que la distinction entre domaine physique et domaine logique est une conséquence de la position de cette substance immobile, ou de l'émergence de la philosophie première. Il est, en effet, remarquable qu'au même moment où Aristote distingue entre deux styles de négations du principe de non-contradiction, l'une purement logique, de mauvaise foi qui est le fait de ceux qui parlent pour parler, l'autre physique, de bonne foi et suscitée par la difficulté de penser le devenir, il cite les physiciens comme les seuls prédécesseurs fondés à examiner l'axiome de non contradiction. Et les physiciens « le faisaient, non sans raison, car ils pensaient être les seuls à étudier la nature dans sa totalité, c'est-à-dire l'être » (1005a 31-33); le philosophe au sens premier prend donc la suite des physiciens et non des dialecticiens, ce qui signifie que le traitement de l'axiome n'est pas, de lui-même, dialectique ou logique. On le comprendra aisément si l'on se souvient que les contraires sont des principes physiques et que l'axiome de non-contradiction est un axiome sur les contraires.

$\mathrm{Ce}$ point a une incidence directe sur les débats qui concernent l'éventuelle différence entre philosophie première et philosophie universelle dont on a pu donner une traduction anachronique en termes de différence entre ontologie et théologie. En réalité, avant l'introduction des causes formelles, les physiciens pouvaient penser traiter de la totalité des principes : la physique était donc philosophie première et prenait pour objet ce qui se nommera des principes communs. C'est avec la révolution socraticoplatonicienne que les genres deviennent des êtres et que le logos acquiert une consistance propre, mais quand le logos acquiert une consistance propre, cela signifie qu'il devient «un des genres des êtres»(Sophiste, 260a), autrement dit qu'il existe, à la manière d'une nature. Ce statut substantiel des logoi, qui induit un statut substantiel pour les universels, est au centre de la critique aristotélicienne qui vise à rendre aux seules substances naturelles le statut de substance, la thèse centrale du livre $\mathrm{Z}$ étant que ni les genres ni les universels ne sont des substances. Le retour de la physique dans les traités métaphysiques signifie donc la destitution des universels comme forme des êtres physiques singuliers, ou encore le refus que les éléments des définitions par lesquelles nous connaissons les êtres physiques, à savoir les genres, soient donnés comme les causes de ces êtres. Tel est le contenu de la critique de la participation des sensibles aux intelligibles.

On remarquera que ce retour de la physique dans les traités métaphysiques n'est rien d'inattendu pour le lecteur du traité des Catégories où Aristote affirme que «si les substances premières n'existaient pas, il serait impossible que quelque chose d'autre existe » (2b6). Le traité entend par «substances premières » les substances physiques individuelles telles que Socrate ou Callias. Les traités métaphysiques ne disent pas autre chose quand ils affirment en Z 16 (1040b 24-41a 5) : 
«En outre, ce qui est un ne saurait être en plusieurs lieux en même temps; au contraire, ce qui est commun se trouve être en plusieurs lieux en même temps, d'où il ressort évidemment qu'aucun des universels n'existe à part, en dehors des singuliers. Pourtant ceux qui soutiennent qu'il y a des formes, d'un côté ont raison de les séparer, s'il est vrai qu'elles sont des substances, mais d'un autre côté ils ont tort parce qu'ils disent que la forme est l'unité d'une multiplicité. [30] La raison en est qu'ils ne peuvent expliquer lesquelles sont des substances de cette sorte, les incorruptibles, en dehors des substances singulières et sensibles; ils les font donc identiques par la forme aux substances corruptibles (car celles-là, nous les connaissons), en ajoutant aux choses sensibles le mot «en soi »: «humain en soi » et «cheval en soi ». [...] Donc rien de ce qui se dit universellement n'est substance et aucune substance n'est formée [5] de substances, c'est évident ».

Dans les traités métaphysiques pas plus que dans les Catégories, les universels ne sont des substances.

Certes, on a beaucoup disserté sur le fait que, précisément, dans les traités métaphysiques à la différence de ce qui se produit dans les Catégories, ce qui s'appelle substance première est la forme et non les individus singuliers. Il n'y aurait un problème consistant que si la forme était un universel ou si l'universel était substance. Or, il est clair que ce n'est pas le cas, car ce qui est forme au sens de substance première c'est l'âme, tandis que l'espèce «homme »n'est pas substance et n'est pas forme, mais un composé de matière et de forme. Le texte le plus clair sur ce point est celui de Z 10 (1035b 14-31) :

«Mais, puisque l'âme des animaux [15] (car c'est la substance de l'être animé) est la substance selon l'énoncé de définition, c'est-à-dire la forme et l'être ce que c'est d'un corps de cette sorte (en tout cas, chaque partie, si elle est bien définie, ne sera pas définie sans sa fonction qui n'existera pas sans la sensation), il s'ensuit que les parties de l'âme, soit toutes, soit certaines, sont antérieures à l'animal <comme un tout> composé (et donc il en va de même de l'animal singulier), [20] tandis que le corps et ses parties sont postérieurs à cette substance ; et ce n'est pas la substance, mais le composé qui se divise en ces parties comme en une matière. [...]. Ni l'être humain ni le cheval ni les termes de cette sorte, prédiqués des êtres singuliers, mais universellement, ne sont une substance ; ils sont un composé formé de cet énoncé-ci et de cette [30] matière-ci, pris comme universels ; le singulier, Socrate, est immédiatement formé de la matière dernière, et il en va de même pour les autres êtres ».

Dans les traités métaphysiques, les universels sont des composés de matière et de forme qui ont une structure tout à fait analogue aux composés singuliers physiques, à la différence que dans le cas des composés universels, la matière est une matière intelligible et non une matière sensible. D'une certaine manière, la notion de matière intelligible (Z 11, 1037a 4) est une nécessité si l'on veut donner un sens strict à la synonymie décrite par le traité des Catégories entre les substances physiques et les substances secondes qui sont dites d'elles. Elle est clairement assumée 
en Z 11 (1037a 5-10) et conduit à soutenir la thèse que la forme est ce qui fonde cette synonymie entre les universels et les singuliers :

«À l'évidence aussi, l'âme est la substance première, le corps est la matière, l'humain ou l'animal sont le composé des deux pris comme universels. «Socrate » et «Coriscos », même s'ils sont âme, ont un double sens, car les uns les considèrent comme âme, les autres comme le composé ; mais, s'ils sont, en un sens simple, cette âme-ci et ce corps-ci, le singulier a le même statut que l' [10] universel ${ }^{8} »$.

Cela conduit Aristote, dans le domaine des universels, à constituer l'ousia qui est forme par des différences qui informent un genre matière, exactement comme l'âme est un système de fonctions qui informent un corps matière. On le voit en $\mathrm{Z} 12$ où la différence dernière est donnée comme la forme et la définition de la chose, c'est-à-dire sa substance (1038a 19-20 ; voir aussi sur ce thème $\mathrm{H}$ 2). Il ne faut donc pas confondre la forme et l'universel, sauf à détruire l'ensemble de la construction aristotélicienne : la forme, c'est-à-dire le système des différences et des fonctions, informe la matière, sensible ou intelligible, mais cette forme ne saurait être un universel qui, par définition, est un composé de forme et de matière. C'est toujours un rapport accidentel qui est décrit entre l'individu et l'universel correspondant :

M 10: 1087a 20: «mais la vue voit, par coïncidence, la couleur universelle [20] parce que cette couleur qu'elle voit est une couleur».

A 1: 981a 18: «or les actions et les générations concernent toutes le singulier, car celui qui soigne ne guérit pas l'humain, sauf par coïncidence, mais Callias ou Socrate ou quelque autre [20] de ceux à qui l'on donne un nom (tôn outô legomenôn ${ }^{9}$ ) de cette manière, qui est par coïncidence un humain ${ }^{10}{ }$.

On pourrait trouver tout à fait étrange que «être humain » soit donné dans un rapport de «coïncidence » avec Socrate ou Callias : la coïncidence est, en effet, la traduction de ce que l'on a l'habitude de rencontrer sous le nom d'accident. Peu importe le nom, le fait est que ce rapport ne saurait être donné comme celui d'un individu à son essence, même s'il s'agit bien de son espèce. On sait certes depuis les Catégories qui s'ouvrent sur la distinction entre «être dit d'un sujet» et «être dans un sujet» que les substances secondes ne sont pas dans leur sujet, autrement dit l'humanité n'est pas dans Socrate ou Callias, mais on peut dire d'eux qu'ils sont des êtres humains. Mais s'ils sont des êtres humains, c'est parce qu'ils sont des animaux

\section{Le statut de composé.}

9. Voir 1015b 28.

10. On peut trouver étrange que le rapport entre un individu et l'espèce à laquelle il appartient soit décrit comme un rapport de coïncidence. Ross rapproche ce passage de M 10, 1087a 19. De fait, le rapport entre le singulier et l'universel est décrit, dans les deux passages, comme un rapport de coïncidence. La difficulté de la question est développée en M 10. On ne peut se contenter d'une version faible du paradoxe qui consisterait à comprendre que le médecin soigne un individu et, par coïncidence, un humain. 
terrestres bipèdes, autrement dit qu'ils sont des corps constitués d'une certaine manière, autrement dit tel type d'âme. L'espèce ou la classe universelle, «être humain », n'est pas la cause du fait que Socrate est un être humain, mais ce qui en est la cause est la forme du corps organique qui est le sien, à savoir son âme. Il faut distinguer entre l'âme comme ensemble de différences ou de traits caractéristiques et l'être humain comme espèce commune à plusieurs individus qui ont des corps semblables parce qu'ils ont le même type d'âme. Bref, c'est l'âme qui est cause et forme au sens d'essence, non la classe universelle. Bien sûr, la classe universelle «être humain » et l'individu, Socrate, Callias ou un autre auront les mêmes traits caractéristiques, sans quoi ce ne serait pas la classe recherchée, mais ce n'est pas par participation à une classe que l'individu est ce qu'il est. La connaissance part toujours des singuliers. On pourrait dire, sous un anachronisme, que la forme est l'âme ou un ensemble de traits, pris en compréhension, qui n'est pas identique à l'espèce envisagée en extension. De manière aristotélicienne, la forme pure et sans matière est composée des différences (liées à des contraires) qui ne dépendent pas de la quantité ou de la matière qu'elles informent que cette matière soit intelligible ou universelle (un genre) ou sensible et singulière. Il faut distinguer entre eidos comme forme et eidos comme espèce. Ce n'est pas toujours le plus facile dans l'exercice de traduction, mais c'est absolument nécessaire si l'on veut respecter le fait que les universels ne sont pas causes et qu'ils ne sont pas non plus substances.

Voilà quelles sont les implications pour la théorie des formes du retour de la physique dans les traités métaphysiques et la raison pour laquelle, j'ai cru pouvoir écrire dans l'introduction à la traduction de la Métaphysique ${ }^{11}$ : «Autrement dit, l'objet propre de la Métaphysique d'Aristote est de réformer la théorie platonicienne de la forme comme substance immobile. La réforme est effectuée quand la substance immobile ou la forme est pensée comme moteur immobile. C'est en fait dans la Physique que l'on trouve la description la plus développée de l'objet des traités métaphysiques: "Mais les principes (arkhai) qui meuvent naturellement (physikôs) sont deux, dont l'un n'est pas naturel ; en effet, il n'a pas en lui-même de principe de mouvement; tel est le cas de ce qui meut sans être mû (kinei mê kinoumenon), comme ce qui est complètement immobile et le premier de tous (to te pantelôs akinêton kai to pantôn prôton) et l'essence (kai to ti esti) et la forme (kai hê morphê), car ils sont une fin et un ce en vue de quoi (telos gar kai hou heneka). De sorte que puisque la nature est en vue de quelque chose (epei hê physis heneka tou), il faut que le physicien connaisse un tel principe" ${ }^{\text {"12 }}$.

On voit qu'il y a une pluralité de moteurs immobiles et qu'ils sont tous pris pour objets d'étude dans les traités métaphysiques. Le physicien doit connaître l'existence d'un tel principe, mais il n'est pas son objet d'étude.

11. Op. cit., note 1, p. 33.

12. Physique, II, 7, 198 a 35-b9. 


\section{Conclusion : Le propre de la philosophie première}

On voit donc que le physicien connait tous les principes (les quatre causes sont connues et énoncées dans les traités physiques et la recherche du livre A dans les traités métaphysiques est manifestement un exercice de vérification et non de découverte) et que la cause motrice fait partie des premières causes, quelle est donc la spécificité de la philosophie première ? On peut partir de points divers pour répondre à cette question, à condition de ne pas oublier que les analyses distinctes convergent par l'affirmation de la primauté de la fin et de l'acte.

Une réponse facile à la question semblerait être celle qui privilégie, pour distinguer la philosophie première de la philosophie seconde, l'aspect logique, voire dialectique, de la philosophie première. On espère avoir montré comment le traitement des logoi, au livre $\mathrm{Z}$, vise à priver les universels du statut de cause. Les universels n'ont pas un statut privilégié, il s'agit d'un mal nécessaire ${ }^{13}$, condition de la connaissance scientifique; mais les universels ne sont ni principes ni causes des êtres. Cette destitution des universels comme causes s'accompagne d'un changement dans la conception de l'être et de l'un : ils ne sont plus des genres suprêmes. L'être et l'un sont eux aussi soumis à la logique de l'équivalence entre le plus déterminé et le plus informé. Ce sont les formes dernières des genres qui constituent l'être et l'un, soit les espèces dernières pour l'être, soit les unités de mesure pour l'un, mais la nature de l'être et de l'un varie en chacune des catégories. Le livre Iota, auquel on accorde généralement trop peu d'importance, est à l'Un ce que le livre Gamma est à l'être. Il y a donc une pluralité irréductible des formes de l'être et de l'un, mais les formes les plus intéressantes de l'être et de l'un sont les plus déterminées et les plus complètes: les plus proches des êtres singuliers. L'indivisible en chaque genre. L'indivisible, c'est ce qui n'a plus de potentialité ou ce dont la puissance a été entièrement actualisée, ce qui n'a plus de matière. On doit appliquer ici aussi le schème de la puissance et de l'acte et l'on voit que la fin (le telos) équivaut à l'état accompli où cesse le processus de division. La logique mise en œuvre est celle de la forme comme moteur immobile.

Si l'on veut emprunter la voie logique, on est ramené à la forme comme fin. Si l'on veut emprunter la voie non physique de la physique, la voie des moteurs immobiles, on reviendra aussi à la forme et à la fin. On pourra seulement s'apercevoir que la cause motrice, au sens strict, vaut surtout dans le monde voué à la génération et à la corruption. Un passage de Z 17 (1041a 28-32) le laisse entendre :

« Ainsi donc, on cherche manifestement la cause, c'est-à-dire l'être ce que c'est, pour le dire d'un point de vue dialectique. C'est chercher, dans certains cas, quelle est la fin, par exemple peut-être dans le cas d'une maison ou d'un lit, [30] et, en d'autres cas, quel est le moteur premier, car c'est aussi une cause.

13. Voir Métaphysique, M, 10. 
Mais on cherche une cause de cette sorte-ci quand il s'agit de génération et de corruption, l'autre cause quand il s'agit aussi de l'être ».

De fait, la préexistence en acte d'une substance individuelle n'a aucune justification dans le monde des astres qui, éternels, n'ont pas été engendrés et ne se corrompront pas. Il en va de même pour le monde dont on sait qu'il est éternel. Dans le monde supralunaire, la cause motrice n'a pas la même fonction que dans le monde sublunaire : elle est cause seulement de mouvement, puisque la seule matière dans ce monde est une matière topique. Par cette voie également, on conclut au privilège de la forme et de la fin, mais il est encore plus clair, dans ces exemples, que la forme n'est pas un universel, puisqu'il s'agit, avec les astres, d'exemplaires uniques dont Aristote montrera qu'ils ne peuvent, de ce fait, être véritablement définis, ce qui ne les empêche pas d'exister (Z 15, 1040a 27-33).

On peut donc conclure que le retour de la physique dans la Métaphysique a surtout conduit à réformer la première philosophie première, la platonicienne : les principes des définitions, genres et espèces, ne sont pas les principes des êtres définis. D'où la nécessité de réintroduire la cause motrice, transformée, comme une cause première. La transformation de la cause motrice se marque par la distinction entre les moteurs mus et les moteurs immobiles. L'ancienne physique décrivait seulement des moteurs mus. Son retour est possible, si on la subordonne à une théorie plus complète qui comprend des moteurs mus et des moteurs immobiles; les moteurs immobiles sont des formes en acte.

On pourrait objecter que ce retour de la physique n'est guère un retour victorieux, puisqu'elle est désormais une «philosophie seconde ». À quoi l'on pourrait répondre que proposer une théorie de la forme comme «moteur immobile » est penser la forme dans une problématique orientée par la question du mouvement. De fait, la théorie modale des formes proposée par Aristote, qui répond aux distinctions entre les ousiai, est le signe que la «métaphysique » ou «philosophie première » se définit toujours relativement à la physique. La spécificité de la philosophie première est alors la théorie de la forme comme acte (energeia), tandis que le physicien étudie les moyens par lesquels une forme passe de la puissance à l'acte. 